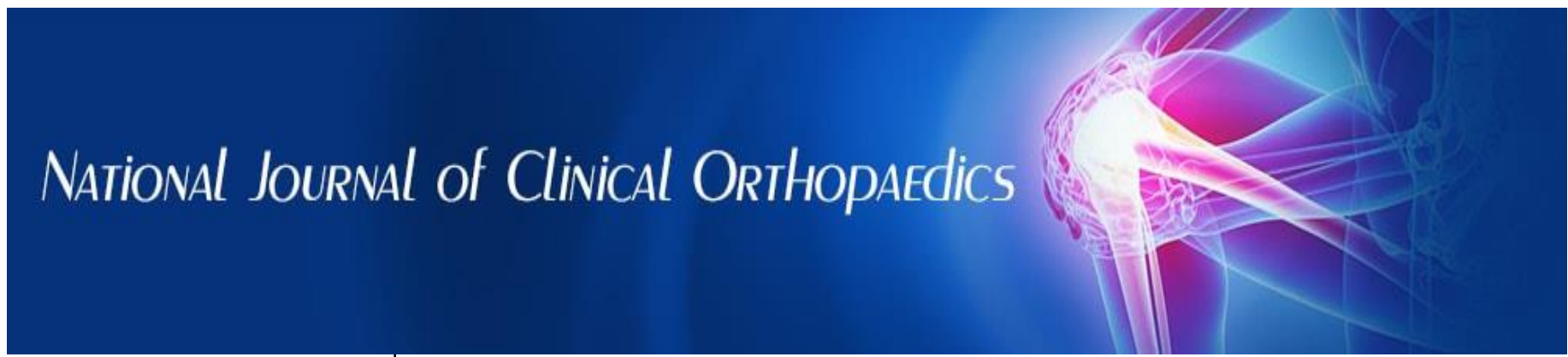

ISSN (P): 2521-3466

ISSN (E): 2521-3474

(C) Clinical Orthopaedics

www.orthoresearchjournal.com

2020; 4(4): 31-34

Received: 05-10-2019

Accepted: 12-11-2019

Dr. Hrishikesh Suresh Saodekar Associate Professor, Department of Orthopedics, Geetanjali Medical College and Hospital, Udaipur, Rajasthan, India

Dr. Kamal Kumar Agarwal Assistant Professor, Department of Orthopedics, Geetanjali Medical College and Hospital, Udaipur, Rajasthan, India
Corresponding Author: Dr. Kamal Kumar Agarwal Assistant Professor, Department of Orthopedics, Geetanjali Medical College and Hospital, Udaipur, Rajasthan, India

\section{Results of proximal femoral nailing in subtrochanteric fractures}

\section{Dr. Hrishikesh Suresh Saodekar and Dr. Kamal Kumar Agarwal}

DOI: https://doi.org/10.33545/orthor.2020.v4.i4a.244

\begin{abstract}
Background and Aim: Subtrochanteric fractures typically occur in the proximal femur between inferior aspect of lesser trochanter and distance of about $5 \mathrm{cms}$ distally or the centre of isthmus of femoral shaft. These fractures of femur are one of the most difficult fractures to treat. The primary aim of the study was to assess the results of proximal femoral nail of subtrochanteric fractures, to assess the functional outcome, radiological union and compare the same with other studies which have used a different modality of treatment.

Material and Methods: This was a retrospective study done on 50 patients with subtrochanteric fractures classified by Sienshiemer classification and operated by proximal femoral nail implant. Clinical evaluation was done by Harris hip score. The data was collected by interviews, follow up at intervals of 1, 2, 4 and 6 months, clinical examination and analyzing case papers.

Results: Most common cause of injury in our study was fall down closely followed by road traffic accident. Associated injury Overall, $24 \%$ of the patients had associated injuries. $17 \%$ of patients had associated injury in form of fracture shaft femur, distal end radius and calcaneum fractures etc. Complications Infection occurred in 3 cases, non-union in 3 cases, Backout of screws in 3 cases and breakage of screws in 2 cases.

Conclusion: The study results conclude that PFN (intramedullary implant) has proved to be better implant than extramedullary implant; PFN is a closed method, thus preserves the fracture hematoma yields early healing and less mean radiological union time in comparison to extramedullary implants; it is a quick procedure in the hands of experienced surgeon who has overcome the 'learning curve' with small incision significant less amount of blood loss and minimal preoperative complications.
\end{abstract}

Keywords: Harris hip score, infection, proximal femur, subtrochanteric fracture

\section{Introduction}

Subtrochanteric fractures account for 7 to $44 \%$ of all proximal femoral fractures ${ }^{[1-3]}$. The inherent instability of the fracture and the enormous muscle forces acting across the fracture fragments render treatment options difficult ${ }^{[4]}$. Often, these fractures are communited. A medial buttress is important to minimise implant stress and fatigue failure ${ }^{[5-7]}$. But when communition is severe, this cannot be achieved. Subtrochanteric fractures typically occur in the proximal femur between inferior aspect of lesser trochanter and distance of about $5 \mathrm{cms}$ distally or the centre of isthmus of femoral shaft. These fractures of femur are one of the most difficult fractures to treat. They represent challenges to achieving stable fixation and appropriate reduction regardless of age, these fractures differ significantly from femoral shaft fractures and more proximal femoral fractures in mechanisms, treatment and complications ${ }^{[8-}$ 10]. The subtrochanteric region is usually exposed to higher stresses during activities of daily living Axial loading forces through the hip joint, create a large lever arm with significant lateral tensile stresses and medial compressive loads. In addition to the bending forces, muscle forces at the hip also create torsional effects that lead to significant rotational shear forces. During normal activities of daily living, up to 6 times the body weight is transmitted to the subtrochanteric region of the femur. As a result of these high forces, the bone in this region is a thick cortical bone with less vascularity and results in increased potential for healing disturbances. Hence, subtrochanteric fracture is difficult to manage and associated with many complications [11]. Options for surgical stabilization of subtrochanteric fractures include dynamic hip screw, gamma nail, proximal femoral nail (PFNs) and proximal femoral plates. 
There are several internal fixation options for managing these fractures that generally fall into two categories: some form of intramedullary fixation or some form of plating. The benefits of proximal femoral nailing (PFN) as an intramedullary implant as per the literature are indirect fracture reduction hence preserving fracture hematoma and decreasing chances of nonunion, lesser complications like blood loss, infection and early mobilization [9]. Intramedullary position of nail acts as mechanical barrier and prevents excessive collapse (controlled collapse) and prevents medialization early ${ }^{[13]}$. Short lever arm provides more force for bending due to intramedullary position. Because of rigid construct it acts as load bearing system and thus can be used in unstable type of proximal femoral fracture also where posteromedial continuity cannot be restored. The antirotation screw in addition to the neck screw provides excellent rotational stability. There is an inbuilt anteversion of the neck screw which helps to prevent retroversion deformity. Also, the anterior bowing and the anteroposterior angle of 60 is anatomical and helps in easy entry of the nail. The laboratory testing elucidated the mechanical performance of the nail in vitro highlighting the advantages and weakness in the design. None of the nails were superior in all tests when compared to PFN ${ }^{[14]}$.

Intramedullary position of implant holds proximal and distal parts in aligned position, restores, limb length and allows fracture to heal in good environment. In comminuted fractures also allows transmission of weight from proximal bony fragment to distal bony fragment (maintaining alignment) thus allows weight to be transmitted through natural bony course. Thus, increases implant life. The primary aim of the study was to assess the results of proximal femoral nail of subtrochanteric fractures, to assess the functional outcome, radiological union and compare the same with other studies which have used a different modality of treatment.

\section{Material and Methods}

This was a retrospective study done on 50 patients with subtrochanteric fractures of femur operated with PFN at tertiary care institute of India, for the duration of 1 year. The data was collected by interviews, follow up at intervals of 1, 2, 4 and 6 months, clinical examination and analyzing case papers. Only fresh Subtrochanteric fractures in adults were included in the study. Pathological fractures, Fractures in children, Old neglected fractures and Peri-prosthetic fractures were excluded from the study. Patient were given spinal or epidural anesthesia and shifted to a radiolucent fracture table in a supine position with perineal post. Operative leg was slightly adducted and put on traction. Opposite limb was put in a full abduction as to give space for the $\mathrm{C}$-arm in between the legs. Reduction was achieved by traction and internal rotation primarily and adduction or abduction as required. Reduction was checked in a $\mathrm{C}$-arm with anterior-posterior and lateral view. A $5 \mathrm{~cm}$ incision was taken above the tip of the greater trochanter and deepened to the gluteus medius muscle. Tip of the greater trochanter palpated and minimal muscle attachment was cleared off. Entry point is taken on the tip of the greater trochanter AP and lateral position. Total time of surgery and blood loss was noted intra operatively. Post-operative protocol Quadriceps physiotherapy, strengthening exercises, SQE and calf pumping are started as soon as the patient is out of anaesthesia, followed by knee and ankle mobilization on post op day 2. Sutures were removed on 12th post-operative day. Patients were advised to walk non weight bearing walking as soon as tolerable usually after suture removal. Partial weight bearing walking was started once further collapse is not expected radiologically around 8 weeks. Full weight bearing walking was allowed after assessing for radiological and clinical union. Patient was discharged around patient oriented discharge. 5 Patient is asked to come for follow up 1, 2, 3 and 6 months from the date of surgery. At each follow up patient is assessed clinically as per Harris hip score (Harris, traumatic arthritis of hip after dislocation and acetabular fractures: treatment by mold arthroplasty. An end result study using a new method of result evaluation-1969 JBJS). X-rays AP/LAT view of hip with femur were taken.

\section{Statistical analysis}

The recorded data was compiled and entered in a spreadsheet computer program (Microsoft Excel 2007) and then exported to data editor page of SPSS version 15 (SPSS Inc., Chicago, Illinois, USA). For all tests, confidence level and level of significance were set at $95 \%$ and $5 \%$ respectively.

\section{Results}

All the cases were treated with intramedullary fixation PFN. In this study all the patients involved were above 17 years of age. The age distribution was from 18 to 87 years of age. In younger and adult injury was caused by high velocity. Out of the 4 young adults who had a low velocity trauma two were of very poor socioeconomic strata and the other two were chronic alcoholic. In elderly age group low velocity trauma causes this fracture. Most of the patients in our study were males. Most common cause of injury in our study was fall down closely followed by road traffic accident. Associated injury Overall, 24\% of the patients had associated injuries. $17 \%$ of patients had associated injury in form of fracture shaft femur, distal end radius and calcaneum fractures etc. five percent of the patients had other system injuries both of them had head injuries. Right extremity was more involved in our study. Overall, most common patterns were type II (two part fracture). Subtype IIIA was the most common individual pattern. Least common pattern seen was type I (UN displaced). Anesthesia In this series most common mode of anaesthesia given was spinal $91 \%$ of the patients with $7 \%$ patients operated under epidural and 3\% under general anesthesia. Operation time Average time of surgery in our series for PFN was 72.30 minutes. The average radiological union time in our series is 5.15 months with 2 non-unions. The average full weight bearing walking time is in our series 4 months. Two patients had nonunion at the end of 9 months followup. In PFN nail 3 patients with long spiral fracture encerclage wiring was done to hold fragments by opening fracture site. In two patients bone grafting was done because of delayed union (Table 3). There was shortening of more than $1 \mathrm{~cm}$ in $22 \%$ patients. Complications Infection occurred in 3 cases, non-union in 3 cases, Backout of screws in 3 cases and breakage of screws in 2 cases.

Table 1: Age or mode of injury

\begin{tabular}{|c|c|c|c|}
\hline Age in years & No of patients & High velocity & Low velocity \\
\hline $18-48$ & $30(60)$ & 26 & 4 \\
\hline$>49$ & $20(40)$ & 4 & 16 \\
\hline Total & $50(100)$ & & \\
\hline
\end{tabular}

Table 2: Distribution of patients as per etiology

\begin{tabular}{|c|c|c|}
\hline Cause & Number & Percentage \\
\hline Road traffic accident & 21 & 42 \\
\hline Fall & 24 & 48 \\
\hline Beaten by opposite party & 5 & 10 \\
\hline Total & 50 & 100 \\
\hline
\end{tabular}


Table 3: Distribution of patients as per etiology

\begin{tabular}{|c|c|c|}
\hline Cause & Number & Percentage \\
\hline Road traffic accident & 21 & 42 \\
\hline Fall & 24 & 48 \\
\hline Beaten by opposite party & 5 & 10 \\
\hline Total & 50 & 100 \\
\hline
\end{tabular}

Table 4: Harris hip score

\begin{tabular}{|c|c|c|}
\hline Results & Number & Percentage \\
\hline Excellent & 20 & 40 \\
\hline Good & 12 & 24 \\
\hline Fair & 11 & 22 \\
\hline Poor & 7 & 14 \\
\hline Total & 50 & 100 \\
\hline
\end{tabular}

\section{Discussion}

Subtrochanteric fractures are usually the result of high-energy trauma and often subjected to significant displacement and great difficulty in close reduction through traction. The high incidence of delayed union, malunion and non-union of fractures has left conservative treatment as advocated by De Lee et al. abolished in modern trauma care ${ }^{[15]}$. Allowing a minimally open approach, intramedullary nailing is closely linked to "biological internal fixation," in addition to its mechanical benefits over plate fixation. Intramedullary fixation allows the surgeon to minimise soft tissue dissection thereby reducing surgical trauma, blood loss, and infection and wound complications [16, 17].

Mean age of the present study population was 47 years and $60 \%$ of them were younger than 49 years. Male predominance was seen in number of cases and most of them belonged to age group of 18-49 years mainly because of more active life and so are more exposed to high velocity trauma. The mode of injury in young is due to a high velocity trauma. The fractures in older population were due to low velocity injuries. This infers that majority of elderly present with low energy osteoporotic fractures. Fractures were classified in the current study according Seinshemer's classification which now is most commonly used classification. Most common type was the II accounting for $35 \%$ of fractures followed by III accounting for $32.5 \%$ fractures. Variables like injury operation interval and operative time and methods and so are subjected to fluctuation. The study stated that fixation with proximal femoral nail takes less time as compared to fixation with other intramedullary and extramedullary devices but results of our study show that there is no significant difference between the two groups in terms of mean duration of surgery. Werner et al. was the first who introduced the term Z-effect, detected in $5(7.1 \%)$ of 70 cases. The incidence of the cut-out of the neck screw in this study was $8.6 \%$. The Z-effect phenomenon is referred as a characteristic sliding of the proximal screws to opposite directions during the postoperative weight-bearing period ${ }^{[18]}$. The most recent study evaluating the use of long PFN is from Fogagnolo et al., who reported 46 patients with an average rate of intraoperative technical or mechanical complications of $23.4 \%$. They also reported 2 implant failures and 1 fracture below the tip of the nail. The AO ASIF in 1996, therefore, developed the long Proximal Femoral Nail to reduce the risk of implant related complications. Therefore, in addition to the $8 \mathrm{~mm}$ load bearing femoral neck screw, the long PFN has a $6.5 \mathrm{~mm}$ anti-rotation screw to increase the rotational stability of the neck fragment. Also minimises stress concentration and tension in the femoral shaft. This should reduce the risk of intraoperative and postoperative femoral shaft fractures. Long PFN also has all the advantages of an intramedullary device such as decreasing the lever arm, can be inserted by closed technique which retains the fracture haematoma, decreases blood loss, minimizes soft tissue dissection and wound infections. In an experimental study, Gotze et al. (1998) compared the loadability of osteosynthesis of subtrochanteric fractures and found that the long PFN could bear the highest loads of all devices ${ }^{[19]}$. Distal locking was always done and patient was mostly allowed bedside hip and knee bending on the 2nd post-operative day and very good patient compliance was seen. Patients were normally discharged after 3rd post-operative day. Prolonged immobilization and nonweight bearing ( $>9$ months) seen in other implants causes significant joint space narrowing ${ }^{[20]} .2$ cases of infection was observed in the study of which only one was deep. In few cases with communition in subtrochanteric fracture encerclage were done to increase the contact at the fracture site, thus increasing the chance of union of fracture. The amount of blood loss during operation was less because the femoral head is not reamed and the fracture site is not exposed compared to other intramedullary implants like Gamma nail or extra medullary implants like dynamic hip screw ${ }^{[21]}$. The average union time in our study was 5.12 months lower than some of the union rates of series with other implants (AO blade plate $7.7 \%)^{[22] . ~ T h e r e ~ w e r e ~} 3$ non unions in our study. Nonunion rate of $28 \%, 10 \%$ for angled plate have been reported by Rahme et al. and Erhan et al. respectively [22, 23]. The fixation of subtrochanterric fractures with intramedullary nail is significantly stronger and more rigid than dynamic condylar screw and dynamic hip screw [17]. Preservation of fracture hematoma, controlled collapse and less chance of post-operative infection aids early fracture union in PFN.

Daniel FA Menzes et al. and Axel Gamulin (2005) ${ }^{[24]}$ in a clinical study of 155 consecutive patients treated with long proximal femoral nail, reported failure of fixation in $2 \%$, femoral shaft in $0.7 \%$, fixation failures included one cut out, one delayed fracture healing and one lateral displacement of the antirotation screw [24]. Simmermacher et al. (1999) ${ }^{[25]}$ in a clinical multicenter study, reported technical failures of the long PFN after poor reduction, malrotation or wrong choice of screws in $5 \%$ of the cases. A cut-out of the neck screw occurred in $0.6 \%$ ${ }^{[25]}$. in our study, we had $4 \%$ failure rate with 1 caseof delayed union.

\section{Conclusion}

Subtrochanteric femoral fractures are usually treated surgically. In the last decade, extra medullary methods of fixation with various angular plates or with a compression hip screw with a plate are more and more replaced by newer intramedullary techniques because of their advantages: The surgical procedure is faster, the blood loss is smaller, the bone healing mainly remains in the reduced position with biomechanically strong fixation, what allows earlier weight bearing on the bone with less local and general complications. Because of increasing occurrence of subtrochanteric fractures in younger age active males, higher demand is placed on treating surgeon to restore near normal function. Osteosynthesis with the long proximal femoral nail offers the advantages of high rotational stability. The study results conclude that PFN (intramedullary implant) has proved to be better implant than extramedullary implant; PFN is a closed method, thus preserves the fracture hematoma yields early healing and less mean radiological union time in comparison to extramedullary implants; it is a quick procedure in the hands of experienced surgeon who has overcome the 'learning curve' with small incision significant less amount of blood loss and minimal preoperative complications. The 
observations of the study found significantly low infection rates and few immediate post-operative complications in PFN; being a minimally invasive technique and operated mostly under spinal anesthesia PFN can be used effectively in elderly patients with multiple pre-existing illness.

\section{Sources of funding: Nil.}

Conflict of interest: None declared.

\section{References}

1. Seinsheimer F. Subtrochanteric fractures of the femur. J Bone Joint Surg Am 1978;60:300-6.2.

2. Craig NJ, Sivaji C, Maffulli N. Subtrochanteric fractures. A review of treatment options. Bull Hosp Jt Dis 2001;60:3546.

3. Sims SH. Subtrochanteric femur fractures. Orthop Clin North Am 2002;33:113-26.

4. Parker MJ, Dutta BK, Sivaji C, Pryor GA. Subtrochanteric fracture of the femur. Injury 1997;28(2):91-5.

5. Kinast C, Bolhofner BR, Mast JW, Ganz R. Subtrochanteric fractures of the femur. Results of treatment with the 95 degrees condylar blade-plate. Clin Orthop Relat Res 1989;238:122-30.

6. Waddell JP. Subtrochanteric fractures of the femur: a review of 130 patients. J Trauma 1979;19:582-92.

7. Watson HK, Campbell RD, Jr Wade PA. Classification, treatment and complications of the adult subtrochanteric fracture. J Trauma 1964;60:457-80.

8. George J, Langford J. Subtrochanteric fractures; Rockwood and Green's Fractures in adults. Volume 11. 7th International edition. Wolters Kluwer 2010.

9. Fielding JW, Magliato HJ. Subtrochanteric fractures. Surg Gyn Obstet 1966;122:555-60.

10. Morihara T, Arai Y, Tokugawa S, Fujita S, Chatani K, Kubo T. Proximal femoral nail for treatment of trochanteric femoral fractures. J Orthop Surg (Hong Kong) 2007;15(3):273-7.

11. McLaurin TM, Lawler EA. Treatment modalities for subtrochanteric fractures in the elderly. Techniques in Orthopaedics 2008;23(3):232-48.

12. Curtis MJ, Jinnah RH, Wilson V, Cunningham BW. Proximal femoral fractures: a biomechanical study to compare intramedullary and extramedullary fixation. Injury 1994;25(2):99-104.

13. Tencer AF. Biomechanics of fixation and fractures; Rockwood And Green's Fractures In Adults. Volume 1. 7th Edition 2010:3-42.

14. Jones T, Kop A, Swarts E, Day R, Morrison D, Keogh C. Bioengineering Bulletin; Department Of Medical Engineering And Physics; Royal Perth Hospital 2008.

15. 4. DeLee JC, Clanton TO, Rockwood CA. Closed treatment of subtrochanteric fractures of the femur in a modified cast brace. J Bone Joint Surg Am 1981;63(5):773-9.

16. Leung KS, So WS, Shen WY et al. Gamma nails and dynamic hip screws for peritrochanteric fractures a randomised prospective study in elderly patients. J Bone Joint Surg Br 1992;74(3):345-51.

17. Hinton R, Smith GS. The association of age, race, and, b sex with the location of proximal femoral fractures in the elderly. J Bone Joint Surg Am 1993;75(5):752-9.

18. Tyllianakis M, Panagopoulos A, Papasimos $\mathrm{S}$ et al. Treatment of extracapsular hip fractures with the proximal femoral nail (PFN): long term results in 45 patients. Acta Orthop Belg 2004;70(5):444-54.
19. Boldin C, Seibert FJ, Fankhauser F et al. The proximal femoral nail-a minimal invasive treatment of unstable proximal femoral fractures: a prospective study of 55 patients with a follow-up of 15 months. Acta Orthop Scand 2003;74(1):53-8.

20. Hung S, Nakamura K. Narrowing of the joint space of the hip after traumatic shortening of femur. J Bone Joint Surg Br 1996;78(5):718-21.

21. Schipper IB, Steyerberg EW, Castelein RM. Treatment of unstable trochanteric fractures: randomized comparison of the gamma nail and the proximal femoral nail. J Rone Joint Surg Br 2004;86:86-94.

22. Yolmaz E, Karakurt L, Güzel H, Serin E. Evaluation of treatment results with the 95-degree AO/ASIF angular platein subtrochanteric femur fractures. Joint Dis Rel Surg 2005;16(1):42-8.

23. Rahme DM, Harris IA. Intramedullary nailing versus fixed angle blade plating for subtrochanteric femoral fractures: a prospective randomised controlled trial. J Orthop Surg 2007;15(3):278-81.

24. Menezes DF, Gamulin A, Noesberger B. Is the proximal femoral nail a suitable implant for treatment of all trochanteric fractures? Clin Orthop Relat Res 2005;439:221-7.

25. Simmermacher RK, Bosch AM, Werken CVD. The AO/ASIF-proximal femoral nail (PFN): a new device for the treatment of unstable proximal femoral fractures. Injury 1999;30(5):327-32. 